\title{
Monte Carlo Study of the Lateral Distribution of Gate Current Density Along the Channel of Submicron LDD MOSFET's
}

\author{
A. HARKAR ${ }^{\mathrm{a}}$, R. W. KELSALL ${ }^{\mathrm{a}, *}$ and J. N. ELLIS ${ }^{\mathrm{b}}$ \\ ${ }^{a}$ Institute of Microwaves and Photonics, School of Electronic and Electrical Engineering, \\ The University of Leeds, Leeds LS2 9JT, UK; \\ ${ }^{\mathrm{b}}$ MITEL Semiconductors, Plymouth, Devon, PL6 7BQ, UK
}

\begin{abstract}
This paper presents the spatial distribution of hot electrons along the channel of a 0.35 micron MOSFET using the full band pseudopotential Monte Carlo simulator DAMOCLES. The important aspect of this investigation is the implementation of a rigorous statistical enhancement technique along the channel of the device, to probe the hot electrons generated along the channel. Simulations have been carried out for different bias points. The results clearly show that the probability of generating electrons with energies sufficient for injection into the oxide layer is significant only at the drain side of the device. It is also observed that the peak gate current occurs exactly just inside the LDD region coincident with the position of maximum lateral electric field.
\end{abstract}

Keywords: Monte Carlo; MOSFET; Statistical enhancement

\section{INTRODUCTION}

With the advancement of microfabrication, chip density goes on increasing and individual transistor size shrinks. Among the various factors that contribute to this remarkable evolution is the development of innovative device structures that minimize the device area and interelemental seperation. This decrease in the critical device dimensions to the 0.1 micron range, accompained by increased substrate doping densities, results in a significant increase in the lateral and vertical electrical fields in the channel region. Under the influence of high lateral fields in submicron MOSFET's, electrons in the channel and pinch-off regions can gain sufficient energy to surmount the $\mathrm{Si} / \mathrm{SiO}_{2}$ energy barrier or tunnel into the oxide. Injection of hot electrons into the gate oxide may result in carrier trapping on defect sites in the oxide, or generation of interface states at the silicon oxide interface, or both. This damage caused by hot carrier injection affects the transistor characteristics, causing a shift in threshold voltage, degradation in transconductance, and a general decrease in the drain current capabilities $[1,2]$. Thus these phenomena constitute a major potential problem for long-term device reliability.

*Corresponding author. Tel.: +44 113 2332068, Fax: +44 113 2332032, e-mail: r.w.kelsall@leeds.ac.uk 
Degradation processes due to hot electrons in silicon devices and circuits have been studied extensively during the past decade and several experimental and simulation studies have been carried out to analyse, understand and model the degradation due to interface state and carrier trapping in the oxide $[3,4]$. So far, however it has not been simple to form a definite picture of the lateral distribution of interface traps or trapped charges in the oxide along the channel [5]. To gain better insight for analysis and modeling of the damage mechanism, as well as associated reliability problems, it is highly desirable to have information about the lateral distribution of interface traps and hot electrons above the damage threshold. However due to limitations of the probing techniques and theoretical models used, the picture is still elusive.

With this motivation the present study was undertaken, involving an investigation of the distribution of electrons with energy greater than $3.3 \mathrm{eV}$ along the channel of the simulated structure, and calculation of the gate current density profile along the channel. Here we present a detailed simulation study of the spatial and energy distributions of hot electrons and their application in modeling gate current density profiles along the channel of 0.35 micron LDD MOSFET's.

\section{DEVICE SIMULATOR}

One of the most powerful methods for investigating hot electron related degradation is an ensemble Monte Carlo simulation including a full band description and a self-consistent calculation of the internal electric field. For this reason we have used the IBM DAMOCLES [6] simulator. DAMOCLES incorporates a pseudopotential bandstructure model which gives accurate information on the high energy electronic states and electron energy distributions. Spatial resolution of the energy distribution places extremely high demands on the sampling capability of the simulation at the high electron energies. The DAMOCLES code address this issue by providing a sampling enhancement (or statistical enhancement) algorithm, and for this work it was essential to define an extensive sampling enhancement scheme.

Thus as a part of this investigation, an extensive sampling enhancement scheme was formulated and implemented in DAMOCLES, which helped to resolve the distribution function over 40 orders of magnitude. In order to incorporate this enhancement technique in DAMOCLES, for the present set of simulations the channel has been spatially divided into distinct regions, and within each spatial region, kinetic energy is partitioned into intervals. Thus the entire span of position, from the source to the drain junction, and kinetic energy space (up to typically $3.8 \mathrm{eV}$ ) in the device is divided into statistical boxes. In each box a different superparticle charge may be assigned. During the simulation, as particles move from common to rare regions in real and energy space, they are subdivided according to these superparticle charge factors, thus increasing the sampling capability in the sparsely populated regions of space.

The spatially localised energy distributions obtained in these boxes were used in conjunction with the spatial distributions of carrier density and normal velocity component to obtain the spatially distributed gate current density profile. The gate current was then modeled as a post processing of the DAMOCLES results as

$$
J_{G}=\int_{\Phi}^{\infty} q n v_{y}(E) f(E) d E
$$

where $\mathrm{q}$ is the electronic charge, $\mathrm{n}$ is the electron concentration, $\mathrm{E}$ is electron kinetic energy and $\Phi$ represents the $\mathrm{Si} / \mathrm{SiO}_{2}$ potential barrier height. $v_{y}(\mathrm{E})$ and $f(\mathrm{E})$ are the normal velocity and energy distribution functions respectively.

\section{RESULTS AND DISCUSSION}

The device structure simulated in this work is based on 0.35 micron conventional LDD CMOS 
technology developed by MITEL semiconductors. The device structure simulated has an LDD junction doping density of $4 \cdot 10^{18} \mathrm{~cm}^{-3}$ and an oxide thickness of $7 \mathrm{~nm}$. The device has channel threshold and punchthrough implants and an ntype polysilicon gate.

Simulations were carried out for two bias conditions. Figure 1 shows the lateral $(\hat{x})$ electric field profiles obtained for $\mathrm{Vd}=\mathrm{Vg}=3.5 \mathrm{~V}$ and $5.0 \mathrm{~V}$ whilst Figure 2 shows the vertical $(\hat{y})$ electric field distribution along the channel of the device. Figures 3 and 4 show the electron energy

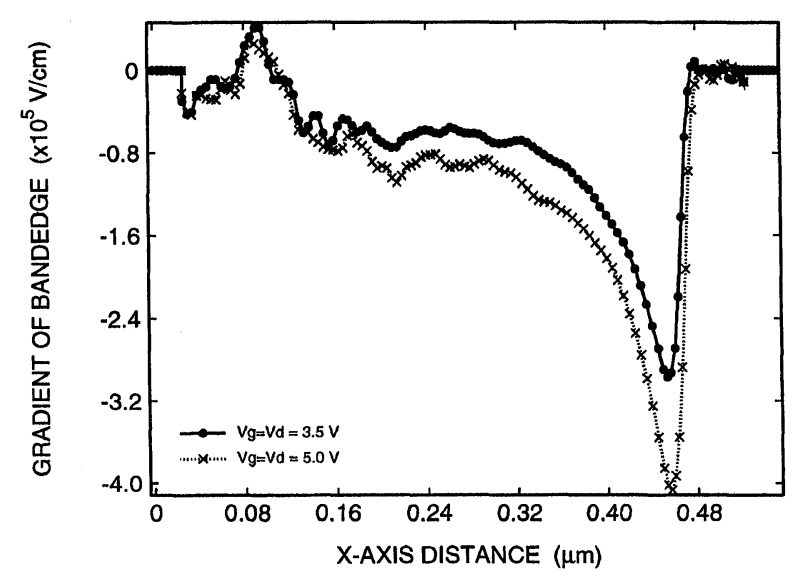

FIGURE 1 Lateral electric field component along the channel.

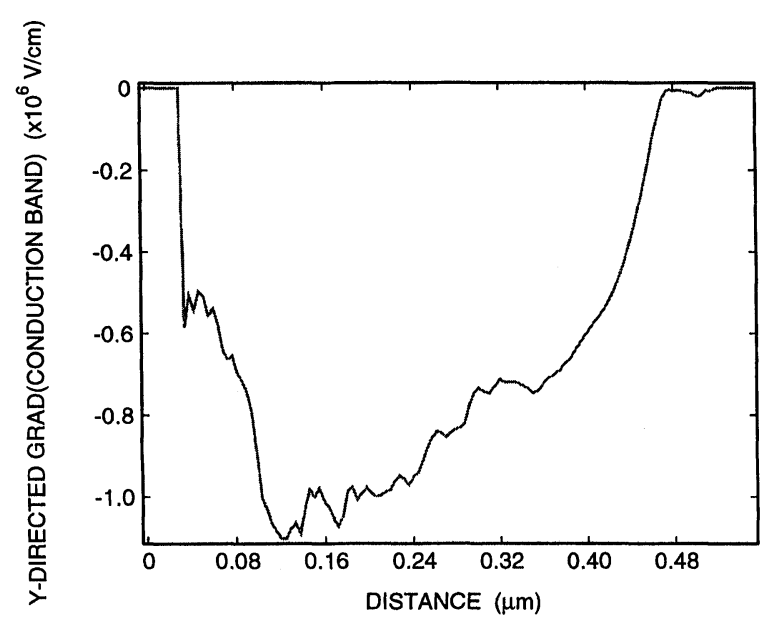

FIGURE 2 Vertical ( $y$ directed) component of electric field along the channel.

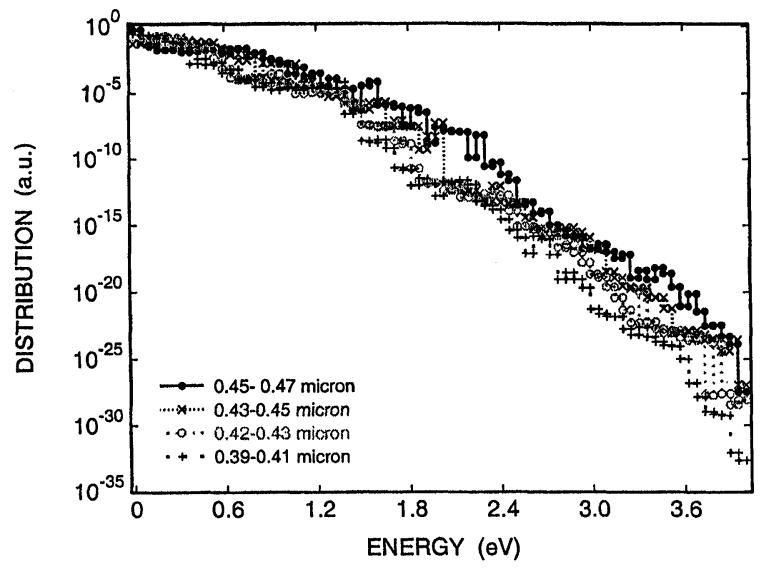

FIGURE 3 Electron energy distribution at different positions along the channel for $\mathrm{Vg}=\mathrm{Vd}=3.5 \mathrm{~V}$.

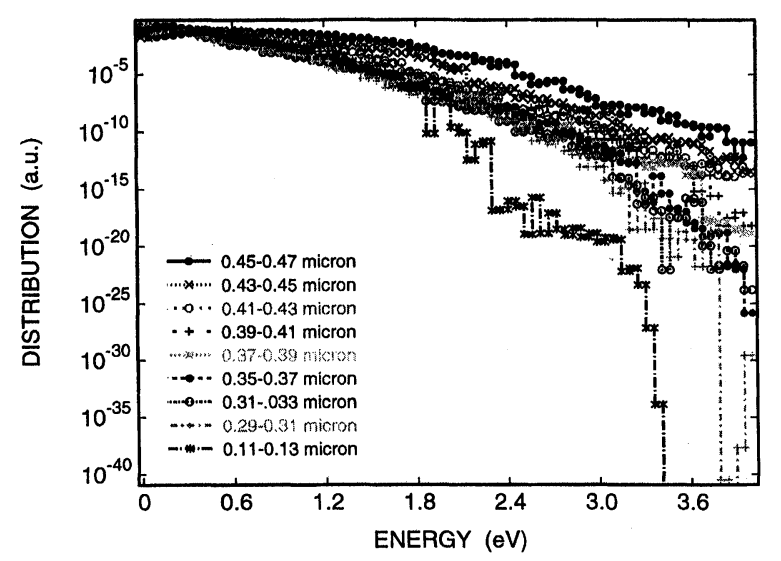

FIGURE 4 Electron energy distribution at different positions along the channel for $\mathrm{Vg}=\mathrm{Vd}=5.0 \mathrm{~V}$.

distribution function at different positions along the channel. From this study the following results are observed: the probability of generating electrons with energies sufficient for injection into the oxide layer $(\geq 3.3 \mathrm{eV})$ is significant only at the drain side of the device. This can be attributed directly to the high lateral electric field in this region. On the other hand, the vertical electric field has little influence on electron heating, since, despite the high vertical field at the source side, no significant hot electron population is observed in this region. It can also be seen that spatial extent of the hot electron region extending from the 


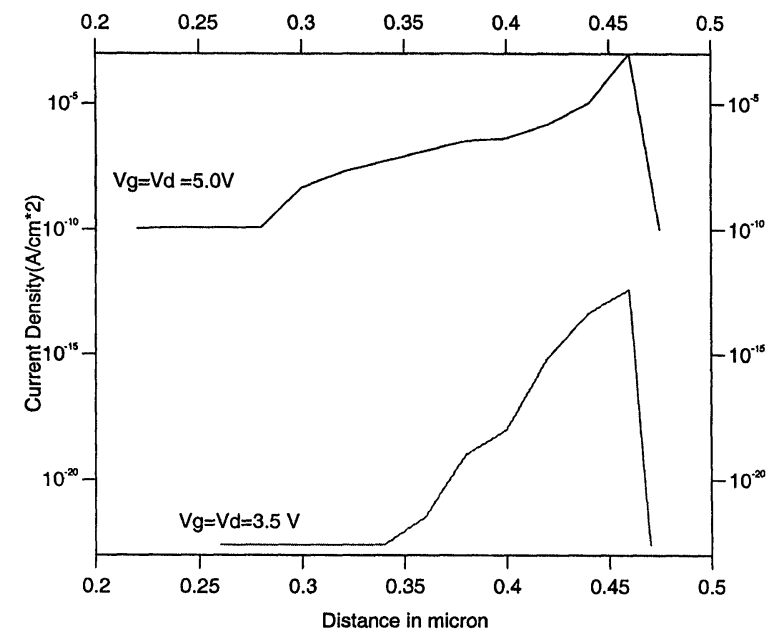

FIGURE 5 Gate current density along the channel.

drain side is greater in case of the higher drain voltage.

The hot electron distribution dominates the form of the gate current density profile along the channel. The peak gate current occurs just inside the LDD region, Figure 5, coincident with the position of maximum lateral electric field. Beyond this point the gate current decreases abruptly corresponding to abrupt falls in both the lateral electric field and hot electron population.

Given the extremely small thickness of the oxide layer $(7 \mathrm{~nm})$ it is reasonable to assume a strong spatial correlation between gate current and hot electron-included interface state generation. Thus it may be concluded that the maximum density of hot electron induced interface states occurs just inside the LDD junction, as has been indicated by some recent experimental work [5].

\section{Acknowledgments}

$\mathrm{AH}$ and RWK would like to thank EPSRC for financial assistance and S. Laux and M. V. Fischetti for useful discussions during the course of this work.

\section{References}

[1] Wang, C. T., Hot Carrier Design Consideration for MOS Devices and Circuits London: Chapman and Hall, 1992.

[2] Ancona, M. G., Saks, N. S. and McCarthy, D., Lateral Distribution of Hot-Carrier Induced Interface Traps in MOSFET's , IEEE Trans on Electron Devices, 35(12), 2221 Dec., 1988.

[3] Wang, T., Huang, C., Chou, P. C., Chung, S. and Tse-En Chang, Effects of Hot Carrier Induced Interface State Generation in Submicron LDD MOSFET's, IEEE Trans. on Electron Devices, 41(9), 1618, Sept., 1994.

[4] Mahapatra, S., Parikh, C. D., Rao, V. R., Viswanathan, C. R. and Vasi, J., A Comprehensive Study of HotCarrier Induced Interface and Oxide Trap Distribution in MOSFET's Using a Novel Charge Pumping Technique, IEEE Trans. Electron Devices, 47, 171, Jan., 2000.

[5] Chung, S. S. and Jiuun-Jer Yang, A New Approach for Characterizing Structure-Dependent Hot-Carrier Effects in Drain Engineered MOSFET's, IEEE Trans. on Electron Devices, 46(7), 1371, July, 1999.

[6] DAMOCLES (2.99) Manual. 

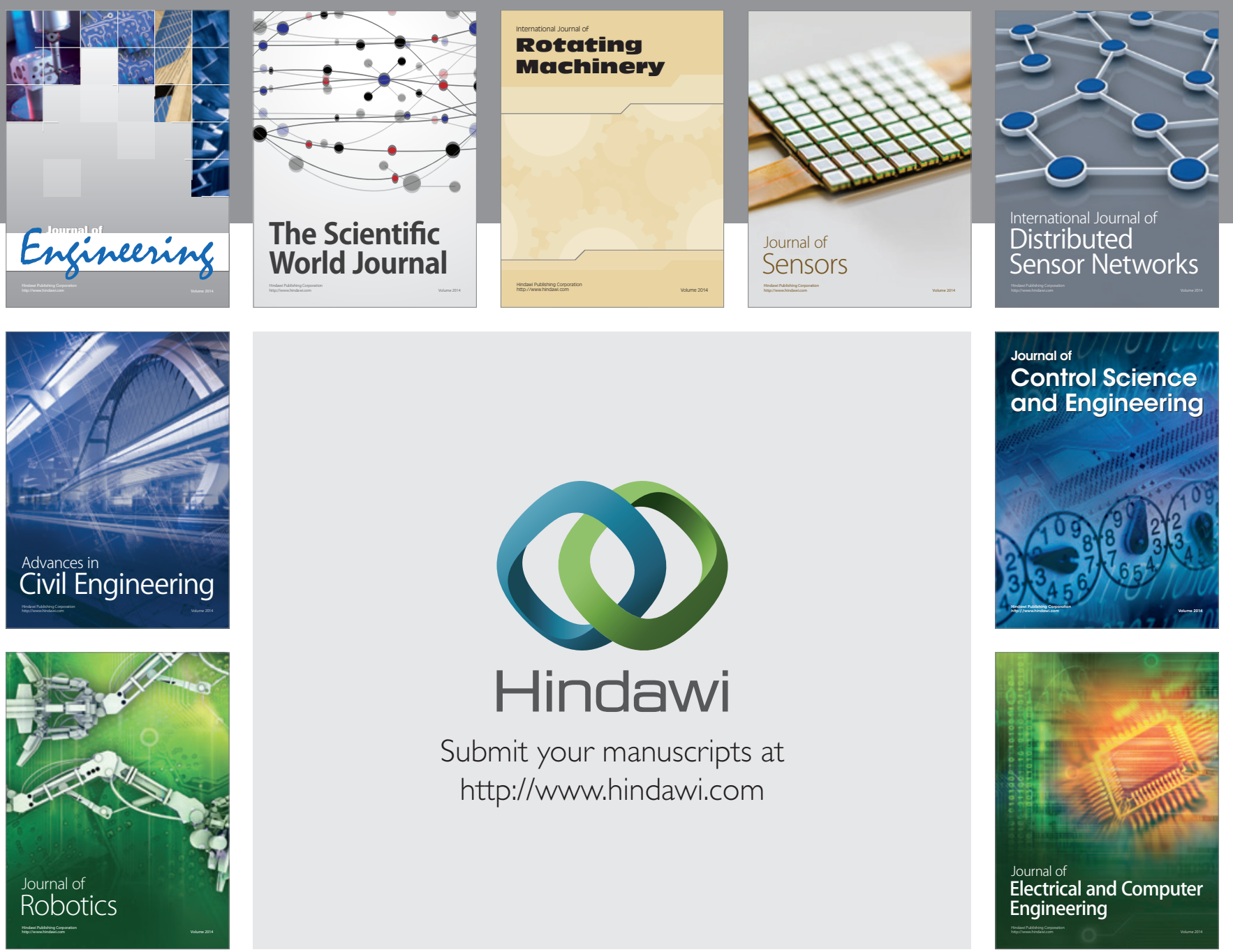

Submit your manuscripts at

http://www.hindawi.com
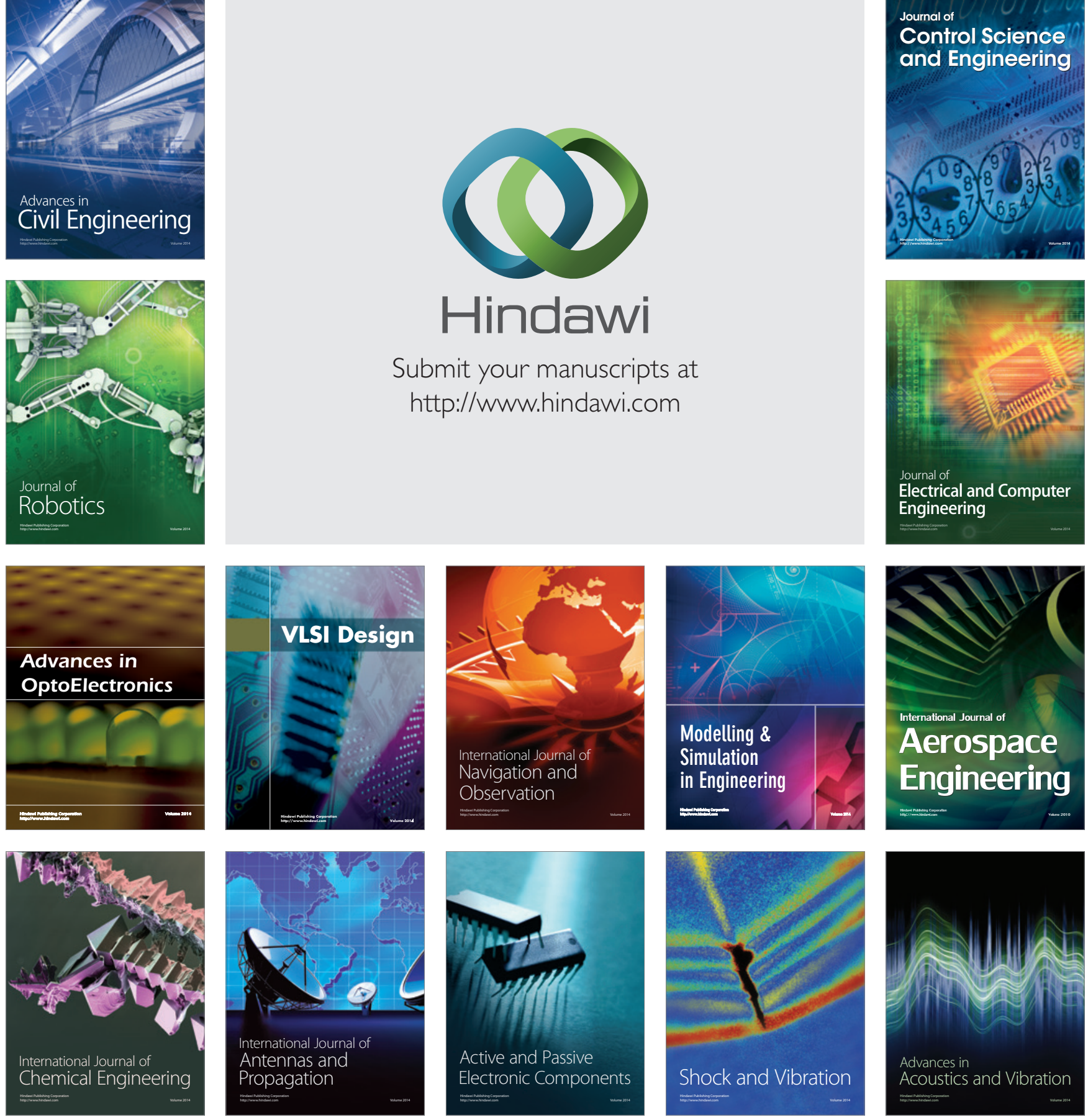